\title{
A influência do cronotipo e da qualidade do sono na frequência de treinamento na academia
}

\section{The influence of chronotype and sleep quality in the frequency of training in the gym}

Rev Bras Ativ Fís Saúde p. 262-269 DO

http://dx.doi.org/10.12820/rbafs.v.20n3p262

1 Universidade Estadual de Campinas, Faculdade de Ciências Aplicadas, Limeira, São Paulo, Brazil

Rodrigo B Siviero

Gabriela F Braga

Andrea M Esteves ${ }^{1}$

\section{RESUMO}

A partir do aumento do número de indivíduos frequentadores de academia nos últimos anos, o aprimoramento entre os horários para a realização de exercícios físicos juntamente com o cronotipo pode ser fundamental para o aumento da motivação diária e melhora das respostas fisiológicas do corpo. Assim, o objetivo do estudo foi analisar a relação entre cronotipo, sonolência e qualidade do sono com horários e frequência de treinamento físico em uma academia da cidade de Limeira. Para isso, 100 frequentadores de uma academia da cidade de Limeira entre 14 e 65 anos, de ambos os sexos, foram avaliados utilizando questionários para determinar o cronotipo, sonolência diurna excessiva e a qualidade do sono. Para controlar a frequência de alunos na academia foi utilizado o banco de dados do Sistema Integrado de Informação da academia (horários de acesso dos alunos). Os resultados demonstraram uma associação significativa entre o cronotipo vespertino e o horário de treinamento em seu período correspondente (noite) $\left(\chi^{2}(1)=12,84, p=0,001\right.$ ), ao passo que os indivíduos matutinos não apresentaram um padrão de horário para o seu treinamento. Não houve relação entre a frequência semanal na academia e o cronotipo $(\mathrm{p}=0.489)$. A qualidade do sono e a sonolência $\left(\chi^{2}(1)=2,31, p=0,195\right)$ não demonstraram associação com o treinamento em período correspondente ao cronotipo. Dessa forma, os resultados sugerem que a qualidade do sono não influenciou na frequência da prática do exercício físico na academia, no entanto, os indivíduos que apresentaram cronotipo vespertino tiveram maior adesão em realizar seu treinamento no período correspondente ao seu cronotipo.

\section{PALAVRAS-CHAVE}

Exercício físico; Sonolência; Ritmo Circadiano; Adesão.

\begin{abstract}
Currently, the growth in the number of individuals in the gym is increasing. The schedules optimization for performing physical exercises along with chronotype can increase the daily motivation and enhance the physiological responses of the body. Thus, the objective of this study was analyzing the relationship between chronotype, sleepiness, and sleep quality with schedule and frequency of physical training in a gym. One bundred participants between 14 and 65 years, both genders, were assessed using questionnaires to determine chronotype, excessive daytime sleepiness and sleep quality. To control the students frequency was used the integrated information system databases from the gym (access schedules of the students). Data analysis was based on the evaluation of the results obtained from the questionnaires, the weekly average frequency and the most common training schedule during the monitoring period. The results showed a significant association between chronotype and evening bours of training in its corresponding period (night) $\left(\chi^{2}(1)=12,84, p=0,001\right)$, while the morning subjects did not present a pattern of time for your training. There was no relationship between the weekly frequency in gym and the chronotype $(p=0.489)$. The quality of sleep and sleepiness $\left(\chi^{2}(1)=\right.$ $2,31, p=0,195)$ showed no association with the training period corresponding to chronotype. Thus, the results suggest that sleep quality did not influence the frequency of physical exercise in the gym; bowever, individuals who exhibited greater adherence evening chronotype had to bold his training in the period corresponding to their chronotype.
\end{abstract}

\section{KEYWORDS}

Physical Exercise; Sleepiness; Circadian Rhythm; Adhesion. 


\section{INTRODUCCÃO}

Os benefícios dos hábitos regulares da prática do exercício físico e do sono saudável têm sido documentados na literatura científica ${ }^{1-4}$. Esses benefícios se estendem para além dos parâmetros físicos, promovendo alterações fisiológicas e psicológicas positivas, melhorando a saúde, conservando funções do corpo e prolongando a vida ${ }^{5,6}$. Apesar disso, na sociedade atual, o que se evidencia é a diminuição progressiva na média da duração do sono e na frequência da prática de exercícios físicos da população em decorrência da falta de tempo ${ }^{8,9}$, culminando em indivíduos sedentários.

No entendimento dessa redução da duração do sono, é de suma importância a avaliação do comportamento dos indivíduos ao longo das horas de vigília, verificando o desempenho nas atividades diárias (físicas e mentais) em função da atuação do "relógio biológico", para que estas sejam mais produtivas, garantindo uma noite de sono de boa qualidade ${ }^{10,11}$.

A prática de exercícios físicos no horário mais adequado pode significar a permanência do praticante dentro do programa de treinamento. Infelizmente, há uma crescente desistência por parte desses praticantes principalmente nos primeiros seis meses a partir do momento em que se inicia a atividade ${ }^{12-14}$.

Para o profissional que trabalha em academias, um dos maiores desafios na retenção de alunos ao treinamento, segundo Liz e colaboradores ${ }^{9}$ é motivar o praticante para que ele venha até a academia e promover resultados a partir de curtas sessões de treino (curtas porque devem se encaixar e respeitar a rotina diária de trabalho, dividindo espaço com outras atividades diárias). Assim, levando esses aspectos em consideração, seria de suma importância encontrar uma forma de conciliar a motivação, o empenho e o tempo disponível de cada indivíduo para prática de exercícios físicos, norteando suas necessidades fisiológicas para que estes aderissem melhor ao treinamento físico.

A literatura já vem demonstrando a relação do sono e do cronotipo (matutino, vespertino e indiferente) influenciando em um melhor rendimento na prática de exercícios físicos, assim como nas suas variáveis ${ }^{15,16}$, porém são escassos os estudos relacionados a horários adequados de treinamento para praticantes de exercício físico (ou dos novos praticantes) em academias de ginástica.

Nesse sentido, o objetivo do presente estudo foi analisar a relação entre cronotipo, sonolência, e qualidade do sono com o horário e a frequência de treinos físicos realizados em uma academia.

\section{MÉTODOS}

\section{Sujeitos}

Participaram deste estudo 100 voluntários (50 homens e 50 mulheres), com idades entre 14 e 65 anos $(32,7 \pm 11,0$ anos), praticantes de atividade física em uma academia de classe média alta da cidade de Limeira-SP, Brasil. A população avaliada representou aproximadamente $15 \%$ dos alunos matriculados na academia. Os voluntários foram acompanhados durante 5 meses nos três períodos de funcionamento do estabelecimento, sendo 30 voluntários (38,4 anos $\pm 13,0$ anos) no período da manhã (06-11horas), 17 voluntários $(29,9$ anos $\pm 13,0$ anos) à tarde (12-17horas), e 53 voluntários (30,4 anos \pm 7,6 anos) no período da noite (18-22 horas), aleatoriamente. Foram incluídos 
no estudo os alunos matriculados no mínimo há um mês na academia, não sendo considerado como critério de exclusão características individuais dos voluntários. Todos os voluntários foram informados sobre os procedimentos do experimento e suas implicações, assinando um Termo de Consentimento Livre e Esclarecido para participação deste estudo. O protocolo do estudo foi aprovado pelo Comitê de Ética em Pesquisa da Faculdade Integradas Einstein de Limeira (nº 231.712).

\section{Desenho experimental}

Com a prévia autorização concedida pelo proprietário-gerente da academia, foi iniciada a seleção de voluntários de forma aleatória, visto que estes foram abordados pelo pesquisador perguntando do interesse em participar do estudo. Os questionários foram respondidos pelos voluntários em um mesmo local disponibilizado pela academia, sob orientação individual.

As coletas de dados foram realizadas apenas por um pesquisador, que ficou responsável pela aplicação dos questionários. No primeiro encontro foram aplicados os questionários de cronotipo, sonolência e qualidade do sono. Os questionários foram entregues aos voluntários e todos receberam a mesma orientação verbal, e as dúvidas foram esclarecidas pelo responsável pela aplicação destes instrumentos. Não houve limite de tempo para preenchê-los. Foi coletada a informação no banco de dados referente ao acesso na academia somente dos voluntários que permaneceram matriculados por 5 meses após responder aos questionários.

\section{Procedimento Experimental}

- Questionário de Cronotipo (Morningness-eveningness Questionnaire-MEQ) O questionário de cronotipo $^{17}$ validado para a população brasileira ${ }^{18}$ foi utilizado para caracterizar a preferência dos sujeitos por serem mais matutinos ou vespertinos. A soma das questões gera uma pontuação que varia 16-86 pontos, com valores mais baixos correspondendo a cronotipo vespertino ${ }^{18}$.

- Questionário de Qualidade do Sono (Pittsburgh Sleep Quality Index - PSQI) O índice de qualidade do sono foi obtido por meio do PSQI de Buysse e colaboradores $^{19}$, já validado e traduzido para a língua portuguesa (PSQI-BR) por Bertolazi ${ }^{20}$, que consiste em 21 itens que avaliam a qualidade do sono e seus distúrbios através do registro do último mês nos componentes: latência do sono, duração do sono, eficiência do sono, distúrbios do sono, uso de medicamentos e disfunções durante o dia. O critério de classificação se baseia na pontuação total obtida, agrupando segundo o sono dos participantes, como bom (abaixo de 4 pontos) ou ruim (igual á 5 ou superior).

- Questionário de Sonolência (Epworth Sleepiness Scale-ESS)

O questionário de sonolência se caracteriza por uma avaliação capaz de diferenciar as pessoas com e sem sonolência daquelas com sonolência excessiva. É composto por 8 (oito) questões que descrevem situações cotidianas que podem induzir à sonolência ${ }^{20},{ }^{21}$.

- Bio System 2.0

Os registros de acesso dos alunos no período da análise foram obtidos através 
do sistema integrado da base dados pela internet (Bio System 2.0), acessível somente com a senha dos colaboradores da academia. Esse sistema registra, no exato momento, os acessos através do reconhecimento biométrico do aluno desde a sua matrícula na academia, tornando-o intransferível. A partir da quantidade de acessos totais, divididos pela quantidade de dias úteis dentro do plano de contrato ativo no período da análise, obteve-se a média de acessos semanais.

\section{Análise estatística}

Os resultados foram descritos por média \pm desvio padrão para os dados quantitativos e frequência absoluta e relativa para os dados categóricos. $\mathrm{O}$ teste de Kolmogorov-Smirnov foi utilizado para avaliar a distribuição dos dados, sendo realizados os testes não paramétricos Mann-Whitney (duas amostras) e Kruskal-Wallis (múltiplas amostras). O teste qui-quadrado foi utilizado para verificar as associações entre as variáveis categóricas. O nível de significância considerado foi de $p<0,05$.

\section{RESULTADOS}

A distribuição da população avaliada segundo o cronotipo de MEQ foi de 38 matutinos, 9 vespertinos e 53 indiferentes, conforme demonstrado na figura 1 .

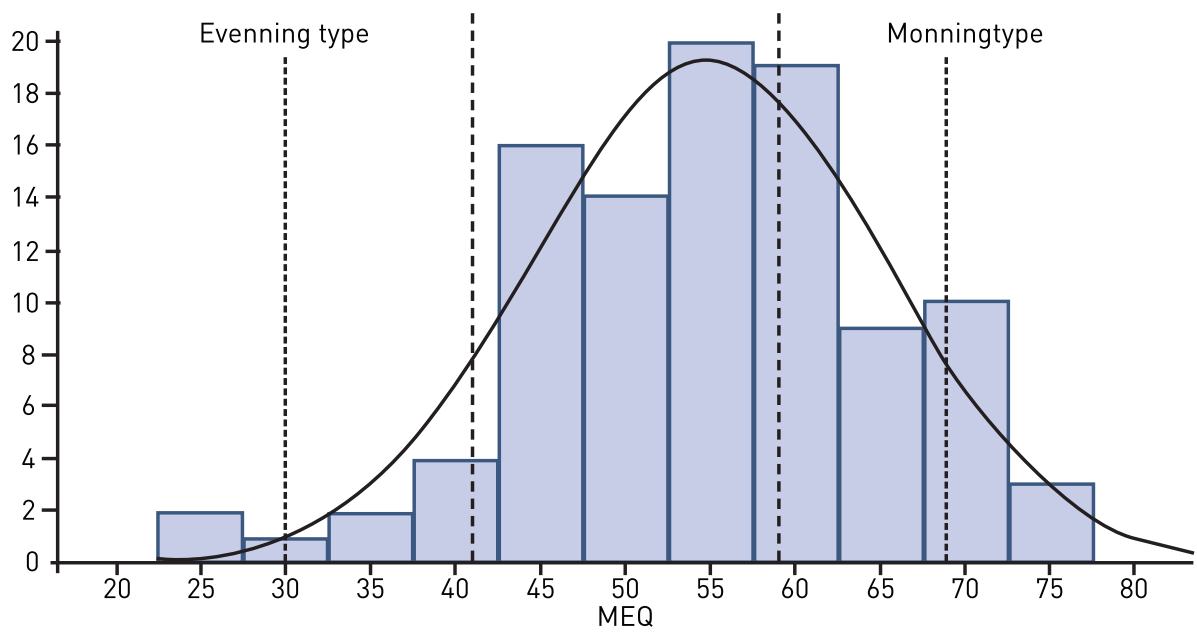

FIGURA 1 - Frequência da distribuição dos voluntários de acordo com o questionário de cronotipo (MEQ). Classificação: vespertino <41, matutino: $>59$ e indiferente $>42<58$.

Houve uma associação significativa entre o cronotipo e treino no horário do cronotipo $\left(\chi^{2}(1)=12,84, p=0,001\right)$. Todos os vespertinos treinaram no horário correspondente ao seu cronotipo e a maioria dos indiferentes apresentou preferência pelo período da tarde/noite. (Tabela 1)

TABELA 1 - Associação entre o cronotipo e período de treino.

\begin{tabular}{lccc}
\hline & & Cronotipo & \\
\hline Período de treino & Indiferente & Matutino & Vespertino \\
\hline Manhã & $11(20,8 \%)$ & $19(50 \%)$ & $0(0 \%)$ \\
\hline Tarde/Noite & $42(79,3 \%)^{*}$ & $19(50 \%)$ & $9(100 \%)^{*}$ \\
\hline
\end{tabular}

*Exato de Fisher (amostras independentes), $p<0,01$ 
Não foi demonstrada diferença estatística nos resultados relacionados à sonolência, cronotipo e horário de treinamento, no entanto $33 \%$ do total da amostra apresentou indicativo de sonolência excessiva diurna (SED).

$\mathrm{Na}$ distribuição da qualidade de sono feita a partir da Escala de Pittsburgh verificou-se que os componentes dividem-se em dois grupos similares (51\% tem uma qualidade de sono ruim, $49 \%$ tem uma qualidade de sono boa). Essa distribuição ao longo dos 3 períodos (manhã, tarde e noite) demonstrou que no período da tarde foi encontrado a maior porcentagem de indivíduos com qualidade de sono ruim (71\%). Não foi encontrado correlação entre os escores obtidos pelas escalas de Epworth e Pittsburgh $(r=0.020 ; p=0.846)$.

Não houve associação entre sonolência e treino no horário do cronotipo $\left(\chi^{2}(1)=2,31, p=0,195\right)$. (Tabela 2$)$

A tabela 3 mostra nos três cronotipos, qual a frequência média semanal de acessos na academia. Foi observado que não houve diferença da frequência semanal entre os cronotipos $(\mathrm{p}=0.489)$.

TABELA 2 - Associação entre sonolência e treino no horário do cronotipo.

\begin{tabular}{lcc}
\hline & \multicolumn{2}{c}{ Sonolência } \\
\hline Treina no horário? & Normal & Sonolência diurna \\
\hline Sim & $22(66,7 \%)$ & $6(42,9 \%)$ \\
\hline Não & $11(33,3 \%)$ & $8(57,1 \%)$ \\
\hline
\end{tabular}

TABELA 3 - Relação do cronotipo com a frequência semanal na academia (dias por semana).

\begin{tabular}{lc}
\hline Cronotipo & Frequência Semanal \\
\hline Indiferente $(n=53)$ & $2,66 \pm 0,18$ \\
\hline matutino $(n=38)$ & $2,87 \pm 0,19$ \\
\hline vespertino $(n=9)$ & $3,22 \pm 0,55$ \\
\hline total $(n=100)$ & $3,07 \pm 0,34$ \\
\hline
\end{tabular}

Nota: Os dados estão apresentados em média \pm erro padrão - Teste Kruskal-Wallis.

Na tabela 4 está apresentado o comparativo da frequência de acessos semanais dos voluntários que treinam dentro do horário relacionado ao cronotipo e os que treinam em outro período fora do indicado. Não houve diferença na frequência semanal entre os indivíduos que treinam no horário do cronotipo e os que não treinam para o grupo matutino. Para o grupo vespertino houve diferença na frequência semanal entre os indivíduos que treinam no horário do cronotipo e os que não treinam ( $\mathrm{p}=0.02)$, uma vez que todos os indivíduos classificados como vespertinos treinavam no horário de seu respectivo cronotipo.

TABELA 4 - Relação da frequência semanal na academia do voluntário que treina no horário respectivo ao cronotipo com o que não treina no horário.

\begin{tabular}{lcc}
\hline Cronotipo & $\begin{array}{c}\text { Trequência Semanal } \\
\text { TREINA no horário } \\
\text { correspondente ao cronotipo }\end{array}$ & $\begin{array}{c}\text { NÃO TREINA no horário } \\
\text { correspondente ao cronotipo }\end{array}$ \\
\hline Matutino $(n=38)$ & $3,10 \pm 0,29$ & $2,63 \pm 0,24$ \\
\hline Vespertino $(n=9)$ & $3,22 \pm 0,55$ & $0^{*}$ \\
\hline
\end{tabular}

Nota: Os dados estão apresentados em média \pm erro padrão. Mann-Whitney $(U=85,50 ; Z=2,31 ; p=0,02)$.

\section{DISCUSSÃO}


Este estudo avaliou a relação entre o cronotipo, hábitos de sono, o horário e a frequência de treinos físicos em academia. O principal achado desse estudo foi que os voluntários do cronotipo matutino são mais flexíveis a se adaptar a outro horário de treinamento, ao passo que os vespertinos apresentam maior rigor em treinar no horário de preferência referente ao seu cronotipo (noite).

$\mathrm{Na}$ atualidade, as pessoas que iniciam o treinamento físico geralmente possuem um tempo restrito para a prática, por isso, procuram adequar o horário para frequentar a academia em função dos afazeres que possuem, e acabam desistindo quando esses horários não atendem de forma adequada as suas necessidades ${ }^{12,15}$.

O ritmo circadiano se comporta de forma diferenciada para cada indivíduo ao longo do dia, sendo importante saber em qual momento ocorre o pico de disposição ou cansaço, para que se possa direcionar e obter o melhor retorno possível de cada sessão de atividade física. As diferenças principais entre matutinos, vespertinos e indiferentes são a preferência de acordar e dormir, as quais têm influencia no desempenho de suas atividades diárias ${ }^{18}$.

Os indivíduos matutinos são considerados mais rígidos quanto ao seu horário de dormir e acordar cedo, o que, no presente estudo, resultou em maiores possibilidades de horários de treinamento (manhã, tarde e noite), diferentemente do grupo vespertino que dormem e acordam mais tarde e assim seguem o padrão de horário referente ao seu cronotipo quando avaliados pelo horário que treinam, pois ficam restritos apenas ao período da tarde e noite para a prática de atividade física.

Em nosso estudo a SED foi observada em 33\% do total de participantes, valor superior ao encontrado por Souza e colaboradores ${ }^{22}(18.9-21.5 \%)$ que avaliaram a sonolência diurna na população de Campo Grande - Mato Grosso do Sul e Ribeirão do Largo - Bahia. Além disso, 51\% da população avaliada apresentou indícios de qualidade de sono ruim, entretanto, esses valores não apresentaram associação com o treinar no horário do seu cronotipo. Alguns estudos sugerem que fatores que a má higiene do sono, de hábitos sociais e de trabalho podem levar há uma restrição do sono e, consequentemente, a SED, que vem associada a vários distúrbios, incluindo a insônia, a depressão, a síndrome da apneia obstrutiva do sono e a narcolepsia ${ }^{23}$. Sem rotinas regulares, ocorre uma desorganização do padrão de sono, afetando-o em quantidade e qualidade, e para os indivíduos, a SED pode significar, dentre outros fatores, prejuízos no desempenho de suas atividades profissionais, sociais e familiares ${ }^{23}$.

Os resultados do presente estudo demonstraram que a qualidade do sono também não influenciou na frequência da prática do exercício físico na academia, apesar dos indivíduos vespertinos apresentarem uma maior adesão em realizar seu treinamento no período correspondente ao seu cronotipo $(100 \%)$.

Pessoas fisicamente ativas têm benefícios quanto à eficiência, ao padrão de sono e à redução na frequência de queixas referentes ao sono, enquanto que pessoas inativas queixam-se de sono ruim, baixa eficiência e são mais estressadas ${ }^{24}$, ${ }^{25}$. Entretanto, a prática deve ser contínua para garantir a manutenção desse estado. Hague e colaboradores ${ }^{25}$ demonstraram em estudo que quando indivíduos treinados sedentarizam-se, mesmo que por um dia, o padrão de ondas e duração dos estágios do sono se redistribui, aumentando a latência do sono de ondas lentas e aumentando o tempo do REM, sem aumentar o tempo total de sono.

O número variado de voluntários nos grupos e a larga faixa etária estudada foi uma limitação para o nosso estudo, visto que o padrão e qualidade do sono 
sofrem alterações com o envelhecimento. Neste contexto, não foi possível verificar se o fator idade estaria influenciando na frequência na academia e no padrão de sono. Entretanto, foi possível sugerir, através de procedimentos estatísticos, as alterações demonstradas na população estudada. Outra possível limitação foi à impossibilidade de utilizar um método padrão-ouro (polissonografia e/ou actigrafia) para a avaliação do padrão e da qualidade do sono. Entretanto a utilização de questionários validados acaba sendo um importante instrumento na pesquisa em diferentes populações, por ser um método mais acessível e menos oneroso. Por fim, os dados analisados foram coletados em apenas uma academia da cidade de Limeira, assim, é importante cautela ao extrapolar os resultados obtidos no presente estudo, visto que fatores culturais, econômicos e sociais também apresentam grande influência no padrão de sono e no perfil dos alunos matriculado em academias.

As orientações e intervenções de exercícios físicos devem ser empreendidas como uma intervenção não farmacológica que apresenta efeitos positivos sobre o ciclo sono-vigília. No entanto estes benefícios ainda são poucos difundidos entre profissionais da área da saúde por ainda não considerarem importante este fator como parte do treinamento físico. Dessa forma se torna importante para o profissional de Educação Física ter conhecimento sobre o cronotipo e a sua influência na melhora do desempenho em diversas atividades, pois com uma otimização das preferências individuais com os horários do dia-a-dia, este profissional ganha um grande aliado no aumento da motivação e melhora do rendimento do seu aluno.

\section{Conflito de interesse}

Os autores declaram não haver qualquer potencial conflito de interesse.

\section{REFERÊNCIAS}

1. Santos RVT, Tufik S, Mello MT. Exercise, sleep and cytokines: is there a relation? Sleep Med Rev. 2007;11:231-39.

2. Chaput JP, Klingenberg L, Sjödin A. Do all sedentary activities lead to weight gain: sleep does not. Curr Opin Clin Nutr Metab Care 2010;13:601-7.

3. Lira FS, Pimentel GD, Santos RVT, Oyama LM, Damaso AR, Oller do Nascimento $\mathrm{CM}$, et al. Exercise training improves sleep and metabolic profile in elderly people in a time dependent manner. Lipids Health Dis. 2011;6:1-6.

4. Viana VA, Esteves AM, Boscolo RA, Grassmann V, Santana MG, Tufik S, et al. The effects of a session of resistance training on sleep patterns in the elderly. Eur J Appl Physiol. 2012;112:2403-08.

5. Kujala UM. Evidence on the effects of exercise therapy in the treatment of chronic disease. Br J Sports Med. 2009;43:8 550-555.

6. Warburton DER, Nicol CW, Bredin SSD. Prescribing exercise as preventive therapy. CMAJ 2006;174: 961-74

7. Bin YS, Marshall NS, Glozier N. Secular trends in adult sleep duration: a systematic review. Sleep Med Rev. 2012;16(3):223-30.

8. Tahara AK, Schwartz GM, Silva KA. Aderência e manutenção da prática de exercícios em academias. Revista Brasileira de Ciência e Movimento 2003;11:7-12.

9. Liz CM, Crocetta TB, Viana MS, Brandt R, Andrade A. Aderência à prática de exercícios físicos em academias de ginástica. Motriz 2010;16:181-8.

10. Peres I, Vetter C, Blautzik J, Reiser M, Pöppel E, Meindl T, et al. Chronotype predicts activity patterns in the neural underpinnings of the motor system during the day. Chronobiol Int. 2011;28(10):883-9. 
11. Squarcini CF, Pires ML, Lopes C, Benedito-Silva AA, Esteves AM, CornelissenGuillaume $\mathrm{G}$, et al. Free-running circadian rhythms of muscle strengh, reaction time and body temperature in totally blind people. Eur J Appl Physiol. 2013;113:157-165.

12. Saba F. Aderência à prática de exercício físico em academias. São Paulo: Manole, 2001.

13. Mcauley E, Morris KS, Motl RW, Hu L, Konopack JF, Elavsky S. Long-term follow-up of physical activity behavior in older adults. Health Psychology 2007;26:375-80.

14. Dishman RK, Buckworth J. Increasing physical activity: a quantitative synthesis. Med Sci Sports Exerc. 1996;28(6):706-19.

15. Halson SL. Sleep in elite athletes and nutritional interventions to enhance sleep. Sports Med. 2014;1:13-23.

16. Youngstedt SD. Effects of exercise on sleep. Clin Sports Med. 2005;24(2):355-65.

17. Horne JA, Ostberg O. A self-assessment questionnaire to determine morningnesseveningness in human circadian rhythm. Int J Chronobiol. 1976;4:97-110.

18. Benedito-Silva AA, Menna-Barreto L, Marques N, Tenreiro S. A self-assessment questionnaire for the determination of morningness-eveningness types in Brazil. In: Hayes DK, Pauly JE, Reiter RJ (Org.). Chronobiology: its role in clinical medicine, general biology and agriculture, part B. New York: Wiley-Liss, 1990.

19. Buysse DJ, Reynolds CF3rd, Monk TH, Berman SR, Kupfer DJ. The Pittsburgh Sleep Quality Index: A new instrument for psychiatric practice and research. Psychiatry Res. 1988;28:193-213.

20. Bertolazi AN. Tradução, adaptação cultural e validação de dois instrumentos de avaliação do sono: Escala de Sonolência de Epworth e Índice de Qualidade de Sono de Pittsburgh. [Dissertação] Porto Alegre: UFRGS;2008.

21. Johns MW. A new method for measuring Daytime Sleepiness: The Epworth Sleepiness Scale. Sleep 1991;14:540-45.

22. Souza JC, Magna LA, Reimão R. Excessive daytime sleepiness in Campo Grande population, Brazil. Arquivos de Neuro-psiquiatria. 2002;60:558-62.

23. Tufik S, Santos-Silva R, Taddei JA, Bittencourt LR. Obstructive sleep apnea syndrome in the Sao Paulo Epidemiologic Sleep Study. Sleep Med. 2010; 11(5):441-6.

24. Flausino NH, Silva Prado JM, Queiroz SS, Tufik S, de Mello MT. Physical exercise performed before bedtime improves the sleep pattern of healthy young good sleepers. Psychophysiology. 2012;49:186-92.

25. Hague JF, Gilbert SS, Burgess HJ, Ferguson SA, Dawson D. A sedentary day: effects on subsequent sleep and body temperatures in trained athletes. Physiol Behav. 2003;78:261-67.

ENDEREC O PARA

CORRESPONDÊNCIA

ANDREA MACULANO ESTEVES

Faculdade de Ciências Aplicadas -

Universidade Estadual de Campinas,

Rua Pedro Zaccaria n. 1300, CEP: $13484-$

350 - Limeira, SP/Brazil.

RECEBIDO 09/03/2015

REVISADO 18/05/2015

E-mail: andrea.estevesafca.unicamp.br 